\title{
BMJ Open Examining mortality risk and rate of ageing among Polish Olympic athletes: a survival follow-up from 1924 to 2012
}

\author{
Yuhui Lin, ${ }^{1,2}$ Antoni Gajewski, ${ }^{3}$ Anna Poznańska ${ }^{4}$
}

To cite: Lin Y, Gajewski A, Poznańska A. Examining mortality risk and rate of ageing among Polish Olympic athletes: a survival follow-up from 1924 to 2012. BMJ Open 2016;6:e010965. doi:10.1136/bmjopen-2015010965

- Prepublication history and additional material is available. To view please visit the journal (http://dx.doi.org/ 10.1136/bmjopen-2015010965).

Received 23 December 2015 Revised 23 March 2016 Accepted 24 March 2016

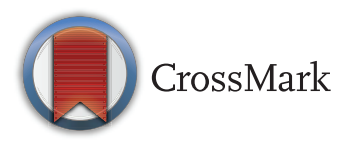

${ }^{1}$ Department of Art and Design Editorial, NaoRococo, Singapore, Singapore ${ }^{2}$ Department of Media Intelligence, Infotech Communications, Media OutReach, Hong Kong, Hong Kong

${ }^{3}$ The Podhale State Higher Vocational School in Nowy Targ, Institute of Tourism and Recreation, Nowy Targ, Poland

${ }^{4}$ National Institute of Public Health-National Institute of Hygiene, Centre for Monitoring and Analyses of Population Health Status, Warsaw, Poland

Correspondence to

Yuhui Lin;

yuhui.linney@gmail.com

\section{ABSTRACT}

Objectives: Population-based studies have shown that an active lifestyle reduces mortality risk. Therefore, it has been a longstanding belief that individuals who engage in frequent exercise will experience a slower rate of ageing. It is uncertain whether this widelyaccepted assumption holds for intense wear-and-tear. Here, using the 88 years survival follow-up data of Polish Olympic athletes, we report for the first time on whether frequent exercise alters the rate of ageing. Design: Longitudinal survival data of male elite Polish athletes who participated in the Olympic Games from year 1924 to 2010 were used. Deaths occurring before the end of World War II were excluded for reliable estimates.

\section{Setting and participants: Recruited male elite} athletes $\mathrm{N}=1273$ were preassigned to two categorical birth cohorts-Cohort I 1890-1919; Cohort II 19201959 - and a parametric frailty survival analysis was conducted. An event-history analysis was also conducted to adjust for medical improvements from year 1920 onwards: Cohort II.

Results: Our findings suggest (1) in Cohort I, for every threefold reduction in mortality risk, the rate of ageing decelerates by $1 \%$; (2) socioeconomic transitions and interventions contribute to a reduction in mortality risk of $29 \%$ for the general population and $50 \%$ for Olympic athletes; (3) an optimum benefit gained for reducing the rate of ageing from competitive sports (Cohort I $0.086(95 \% \mathrm{Cl} 0.047$ to 0.157$)$ and Cohort II 0.085 (95\% Cl 0.050 to 0.144$))$.

Conclusions: This study further suggests that intensive physical training during youth should be considered as a factor to improve ageing and mortality risk parameters.

\section{INTRODUCTION}

A sedentary lifestyle is associated with the onset of chronic diseases including ischaemic heart disease, type-II diabetes and neurodegenerative diseases. ${ }^{1} 2$ In recent studies, obesity among adolescents has been considered as an attributable risk factor for longterm morbidity and premature death. ${ }^{3}$ Frequent exercise is perceived as a major

\section{Strengths and limitations of this study}

- Though the sample size is small, the rate of ageing $\mathrm{d}(\log (\mu(\mathrm{x}))) / \mathrm{dx}$, risk for mortality, and unobserved heterogeneity of the Polish Olympic athletes and the general population, were obtained and presented using the parametric frailty analysis: $y$-Gompertz model.

- The Polish Olympic athletes' 88-year survival data contribute towards one of the longest survival data sets in epidemiological and sports and physical activity studies, and this study has also considered mortality improvements from year 1924 to 2012 and by birth cohorts 1890-1959.

- To avoid the issue of gender-effects in sports performance and survival analysis, and the small sample size of female elite athletes, only male elite athletes are included in the survival analysis.

- The Polish general population and elite athletes born in Cohort I experienced no medical improvement, and are thereby a reliable comparison for mortality risk and on the rate of ageing; for every threefold risk reduction in mortality, the rate of ageing decelerates by $1 \%$.

- A reliable parametric model to account for continuous medical progress is required to adjust for estimates in the Cohort II general population, that is, cohorts born after the year 1920 .

behavioural determinant for improved life expectancy and a slower rate of ageing. There is little doubt that frequent exercise is beneficial for individuals' well-being, and an active lifestyle reduces the risk for chronic diseases. ${ }^{4}$ However, it is still uncertain whether the rate of ageing $d(\log (\mu(x))) / d x$ decelerates in response to frequent and intense physical exercise. The characteristics of sports undertaken (eg, summer or winter sports, endurance or speed-based, amount of energy expenditure) have been extensively studied in previous reports. ${ }^{5-7}$ Our attempt is the first empirical study to show the application of a parametric frailty survival model to gain insights into the rate of ageing and mortality risk for Olympic athletes. This 
analytical work also serves as an example of using survival information of members of a population when no covariate has been recorded, to perform the classic survival analysis approach.

In demographic and actuarial studies, the rate of ageing is usually defined as the relative derivative of mortality $\mathrm{d}(\log (\mu(\mathrm{x}))) / \mathrm{dx}$, and can generally be obtained from the Gompertz hazard function: $\mu(\mathrm{x})=\mathrm{a}$ ' $\exp (\mathrm{bx}) .{ }^{8}$ Therefore, the Gompertz b parameter represents the rate of change in mortality with increasing age $\mathrm{x}$ and we hereafter refer to it as the rate of ageing. This exponentially increasing hazard function is similar to the probability of death $q(x)$, but at older ages $\mu(x)$ values may exceed 1.00. The semilogarithmic graph of mortality rate versus age has been applied for useful inference in many demographic studies, and it is implemented in this study, to recognise the steepness of the hazard gradient as the rate of ageing: that is, $\log \mu(\mathrm{x})=$ $\log (a)+b x$. The y-intercept $\log (a)$ represents the magnitude of the initial hazard at a given age $\mathrm{x}$. As a general illustration, epidemiological studies often compare the hazard risks of two groups: a control and a risk exposed group; online supplementary figure $\mathrm{S} 1 \mathrm{i}-\mathrm{iv}$. If the rate of ageing differs between the groups, the two hazard straight lines will either diverge or converge with increasing age on the semilogarithmic graph. If the rate of ageing is similar, a proportional hazard would be observed and the hazard lines would be parallel.

\section{METHODS}

\section{Data conditioning}

Our participants for this parametric frailty survival analysis were Polish athletes who had participated in the Olympic Games from 1924 to 2010. We assumed that these athletes were elite in their preferred sports expertise, and that they were engaged in frequent, if not intense, physical exercise. The earliest recorded year of birth was 1875, and the latest was in 1982; total $\mathrm{N}=2305$; male $=1828$, female $=477$. For reliable estimates, mortality improvements by calendar events and birth cohort had to be taken into consideration to account for the advancements made in medicine and technology. Recruitment for the Olympic Games was interrupted during the two world wars, WWI and WWII. Therefore, to avoid unreliable mortality risk estimation from war casualties, deaths occurring prior to 1 January 1946 were excluded.

After the consideration of mortality improvements and the statistical power for parametric survival analysis, we restricted our analysis to male athletes born from 1890 to $1959(\mathrm{M}=1273)$. We excluded the small number of deaths of elite athletes born after $1959\left(\mathrm{~N}_{\text {died }}=4\right)$, and the few recruited athletes born prior to year 1890 $(\mathrm{N}=5)$, because they did not serve meaningful interpretation during the survival analysis. The last survival update was on 30 May 2012, when $73 \%$ of male elite athletes were reported to be alive.

\section{Survival analysis}

The shape of the parametric Gompertz hazard function best fits mortality data from age 30 years onwards. The occurrence of excess deaths from risk-taking behaviours during early survival time analysis has to be considered, and a constant term c, commonly referred to as Makeham's c, was included in the Gompertz equation. ${ }^{9}$ In most epidemiological studies, covariates are commonly included during survival analyses, to account for observed or measured heterogeneity in the population. However, there are usually variables left unobserved that may potentially influence mortality risk, for example, region of birth, energy expenditure, socioeconomic status and nutritional intake. We consider these factors as unobserved heterogeneity ( $\gamma$; variance), and assume a $\gamma$ distributed random variable in this study. We implemented (1) the classic Gompertz, (2) Gompertz-Makeham, (3) $\gamma$-Gompertz and (4) $\gamma$-Gompertz Makeham models. In all models, the year of birth was grouped into 10 -year bands, and was considered as a covariate. The most suitable Gompertz hazard model is presented in this article.

\section{The rate of ageing, $d(\log (\mu(x))) / d x$}

The mortality rate of the population is represented as $\mu$ (x), and it has been shown that the Gompertz hazard function fits human population mortality dynamics best: $\mu(\mathrm{x})=\mathrm{a} \times \exp (\mathrm{bx})$. When logarithm is introduced to the exponential increasing hazard, a linear equation is observed: $\log (\mu(\mathrm{x}))=\log \mathrm{a}+\mathrm{bx}$, which represents $\mathrm{a}$ straight line on the semilogarithmic plot: ref Calculus, $y=c+m x$. The rate of change is denoted as $d y / d x$. Hence, to obtain the rate of ageing, given $\mathrm{x}$ is age at death, $d(\log (\mu(x))) / d x=b$.

In populations with unobserved heterogeneity, frail individuals will tend to perish before robust individuals, and lead to selection effects. We assume a $\gamma$ distribution $\mathrm{Z}(\mathrm{x})$ for this mortality selection process: frailer individuals will have a Z-value $>1$ while more robust individuals will have a Z-value $<1$. The mathematical approach to selecting individuals to expire from a population is to divide the population hazard function, therefore a denominator is included to the Gompertz hazard function. The baseline hazard, where $\mathrm{Z}=1$, is equivalent to the standard Gompertz function, see online supplementary equation $\mathrm{S} 1$ and $\mathrm{S} 3$.

To allow us to consider mortality improvements over calendar time, each survival record was split at specified calendar decennial intervals: 1946-1955, 1956-1965, 1966-1975, 1976-1985, 1986-1995, 1996-2005, 20062012. This is considered as an event-history survival analysis (EHA) whereby the survival status of each athlete was tracked at each calendar interval. We fitted our parametric models to mortality schedules of elite athletes born in the two preassigned birth cohorts: 1890-1919 (Cohort I) and 1920-1959 (Cohort II). Mortality risk is influenced by interventions through calendar time and by sociodemographic factors, for example, post war 
living conditions, vaccination coverage rates, education and medical breakthroughs. There is an extensive amount of literature highlighting the contribution of these kinds of sociodemographic factors on life expectancy since the 1890s in Europe. To account for these changes, we used the age-specific mortality trajectories of the general male Polish population as a reference. We accomplished this by fitting Gompertz hazard models to the life-table mortality estimates for Polish males (data source Human Mortality Database, HMD) ${ }^{10}{ }^{10}$ For reliable comparative analysis in the cohort, the standard individual hazard adjusted for unobserved heterogeneity was used to obtain the risk ratio and the percentage change in the rate of ageing (see online supplementary equation S1 and S2). All parameter estimates were obtained using Maximum Likelihood Estimation (MLE) and all estimates presented in this study reached convergence. Age at debut (hereafter, age at recruitment), treated as left-truncation, and vital status, treated as right censoring, for each recruited athlete were introduced during the survival analysis (see online supplementary equation S3). We assumed that athletes received professional training in their sports expertise prior to their debut at the Olympic Games. All analyses were conducted using R-software V.3.0.3.

\section{Research ethics and origin of the Polish Olympics athletes data}

Data collection was initiated by Professor Gajewski in 1993 to provide researchers with the opportunity to conduct studies on elite athletes. The survival profiles of elite athletes who have represented Poland in the Olympic Games were retrieved from the archives of the Polish Olympic Committee, and each survival profile was then cross-validated and supplemented with information from encyclopaedic publications and sports media. Interviews were also conducted with the athletes or with members of their families. Recruitment of Polish Olympic athletes is continuous in this study. Archival death records were verified with the International Society of Olympic Historians.

The study was approved by the Ethics Committee of Jozef Pilsudski University of Physical Education in Warsaw. The database is systematically updated using publicly available sources. Sports press and web-pages are constantly monitored in search of relevant obituary notices. Currently, data collection and survival follow-up of Polish Olympic athletes are performed at the Centre for Monitoring and Analyses of Population Health Status of the National Institute of Public Health in Warsaw.

\section{RESULTS}

From the HMD Poland data source, we observed a decline in age-specific mortality rates for cohorts born from year 1920 onwards; online supplementary figure S2. Prior to the 1920 cohort, the observed $\log \mu(70)$ in the Polish general population suggests little evidence for
Table 1 Descriptive analysis of Polish Olympic Male Athletes born from 1890 to 1959

\begin{tabular}{lccc}
\hline & Cohort & Cohort & \\
& I & II & All \\
\hline Survival Status & 0 & 784 & 784 \\
$\quad$ Alive & 209 & 280 & 489 \\
$\quad$ Dead & 74.6 & 62.8 & 67.8 \\
Age at death (mean) & 26.0 & 24.0 & 24.0 \\
Age at recruitment & & & \\
(median)† & & & \\
Cohort I: 1890-1919; Cohort II: 1920-1959; All: 1890-1959. \\
†Right-tailed distribution (see online supplementary figure S3).
\end{tabular}

mortality improvement. For reliable estimates, we preassigned recruited athletes into two categorical cohorts: 1890-1919 (Cohort I); 1920-1959 (Cohort II), table 1. Cohort I presents a complete case (ie, no censoring). The mean age of surviving athletes at last survival follow-up for Cohort II is at 65.3 years.

The finalised survival analysis considered mortality improvements in the observed survival time. Each recruited athlete survival profile was split in accordance with decennial calendar time intervals. This statistical approach incorporates the survival status of each athlete at each calendar interval during MLE. The advancements in medicine and technology, and changes in social policies that benefit the elite athletes, will hence be accounted for. The obtained estimates suggest that elite athletes enjoy a lower mortality risk than the general population does (figure 1 and table 2). Apart from frequent and intense exercise, the stringent dietary requirements and prioritised access to medical treatments among elite athletes may have accounted for this phenomenon.

\section{Cohort I: individuals born before 1920}

From the $\gamma$-Gompertz model, the estimated rate of ageing b parameter suggests elite athletes born before 1920 experienced a lower rate of ageing than the general population (table 2). Estimates obtained from MLE also suggest that these athletes experienced a threefold risk reduction in mortality accompanied by a $1 \%$ deceleration in the rate of ageing (figure 1, table 2 , see online supplementary equation S3). Individuals born before 1920 did not experience mortality reduction over calendar time (see online supplementary figure S2), hence it is an ideal cohort for a clear insight into whether frequent exercise alters the human rate of ageing. In relation to the relative risk for initial hazard at a given age, athletes enjoy a lower risk for mortality than the general population does (figure 1). Although elite athletes are a distinct occupational group in the population, the estimated $\gamma$ parameter suggests unobserved heterogeneity does exist in this presumed homogenous population. The differences in physiological and energy demands attributed to their frequent 


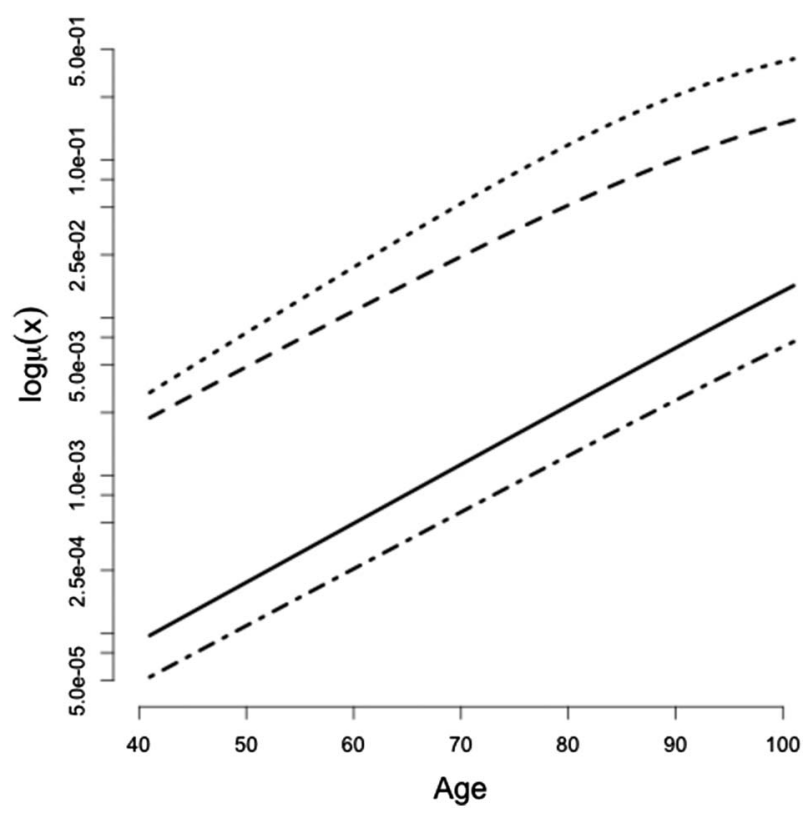

Figure 1 Mortality rates for two birth cohorts on a semilogarithmic plot. The estimates were based on the $\gamma$-Gompertz survival framework. Polish males in the general population (Cohort I, dotted; Cohort II, short dashed); Polish elite athletes (Cohort I, solid; Cohort II, alternating dashed lines). Cohort I representing year 1890 to 1919 , and Cohort II from year 1920 to 1959 .

engagement in competitive sports could have altered their susceptibilities for types of morbidity, a higher likelihood for specific-causes of death and, hence, a higher $\gamma$ among elite athletes than the estimated $\gamma$ in the general population.

\section{Cohort II: individuals born from 1920-1959}

The mortality risk between the general population and elite athletes is shown to be consistent with Cohort I, where athletes experience a lower mortality risk, and hence a longer life-expectancy, than that of the general population. In Cohort II, the estimated rate of ageing is highly similar between elite athletes and the general population, which contradicts our estimates for Cohort I: lower rate of ageing among elite athletes. This may be attributed to mortality improvements from year 1920 onwards in Poland. Interestingly, the comparison of the rate of ageing of elite athletes in Cohort I and II shows a similar rate of ageing (table 2; estimates of elite athletes only). Though our statistical power is low in this study, the Gompertz b estimates suggest the rate of ageing is reduced in Olympic athletes and an optimum benefit gained from competitive sports, reducing the rate of ageing.

Progress made in medical interventions and socioeconomic transitions were also adjusted for during the EHA analysis, to obtain reliable parameter estimates. Among the elite athletes, the estimates suggest that Cohort II individuals benefited from a 50\% mortality risk reduction as compared with individuals born in
Table 2 The $\gamma$-Gompertz estimates for Polish Olympic Athletes and the general population born from 1890 to 1959

\begin{tabular}{|c|c|c|c|}
\hline & \multirow[b]{2}{*}{ Estimate } & \multicolumn{2}{|l|}{$95 \% \mathrm{Cl}$} \\
\hline & & Lower & Upper \\
\hline \multicolumn{4}{|c|}{ Cohort I: 1890-1919 } \\
\hline \multicolumn{4}{|c|}{ Olympic athletes } \\
\hline a & 0.00010 & 0.00000 & 0.38284 \\
\hline $\mathrm{b}$ & 0.08616 & 0.04732 & 0.15687 \\
\hline$\gamma$ & 0.34274 & 0.00162 & 72.65723 \\
\hline \multicolumn{4}{|c|}{ General population } \\
\hline a & 0.00276 & 0.00266 & 0.00285 \\
\hline b & 0.09762 & 0.09707 & 0.09817 \\
\hline$\gamma$ & 0.14099 & 0.13579 & 0.14640 \\
\hline \multicolumn{4}{|c|}{ Cohort II: 1920-1959 } \\
\hline \multicolumn{4}{|c|}{ Olympic athletes } \\
\hline a & 0.00005 & 0.00000 & 0.03921 \\
\hline b & 0.08467 & 0.04995 & 0.14352 \\
\hline$\gamma$ & 1.15127 & 0.54109 & 2.44959 \\
\hline \multicolumn{4}{|c|}{ General population } \\
\hline a & 0.00196 & 0.00192 & 0.00201 \\
\hline b & 0.08327 & 0.08282 & 0.08371 \\
\hline$\gamma$ & 0.22621 & 0.21741 & 0.23536 \\
\hline \multicolumn{4}{|c|}{$\begin{array}{l}\text { The estimates were obtained from MLE and were expressed to } \\
\text { the nearest five decimal place. Parameters: a represents the } \\
\text { magnitude of the hazard; } b \text { represents the rate of ageing; } \gamma \\
\text { represents unobserved heterogeneity. }\end{array}$} \\
\hline
\end{tabular}

Cohort I. The estimated overall mortality risk of the Polish general population is $29 \%$ lower in Cohort II than in I.

\section{DISCUSSION}

Frequent exercise is a fundamental lifestyle habit for reducing risk for morbidity and to increase life expectancy. Compared to the general population, elite athletes are exposed to particularly intense and frequent competing physical exercise, strict dietary requirements and high psychological demands. If frequent exercise is beneficial for survival and decelerates the rate of ageing, then we might expect prominent differences in mortality trajectories between elite athletes and the general population, as shown in figure 1.

As shown in previous studies, the standardised mortality ratio (SMR) derived from the observed/expected mortality of elite athletes to the general population is $<1 .^{11-13}$ This study supports the aforementioned findings as the magnitude of the hazard among elite athletes is much lower than that of the general population, refer to table 2, Gompertz a. Although SMR has been extensively applied in many epidemiological studies, parametric frailty survival analysis provides parameter estimates for hazard risk ratio, and an overview of the age-specific mortality trajectories comprising the rate of ageing and unobserved heterogeneity. We have included Polish males from the HMD, to illustrate the changes in mortality risk that arise from socioeconomic factors for 
mortality at age 70 years. The reconstitution of Poland occurred in 1918, and there was little or no observed mortality progress before 1920. Nevertheless, based on geographic regions and birth cohorts, members of the population who were living in the territory were following the mortality trends of neighbouring regions, presently known as the Slovak Republic and Germany-East and West regions (see online supplementary figure S2). After the 1918 reconstitution, there were improvements in socioeconomic and health policies. Our results suggest the introduction of health policies contributed to the lower observed mortality and a deceleration in the rate of ageing of the population. From the observed population, hazard at age 70 years $(\mu(70))$ indicates that elite athletes have to be categorised into two cohorts to account for socioeconomic changes and medical interventions across calendar time and to obtain reliable mortality estimates.

From the Gompertz frailty survival analyses, we have shown the rate of ageing of Olympic athletes in two preassigned cohorts: without and with medical progress (figure 1 and table 2). In humans, the observed mortality rate has been well-described as an increasing exponential hazard function after young adulthood. ${ }^{8}$ 14-16 We assume elite athletes who have participated in the Olympic Games to be passionate and serious in their expertise to commit to sports as a career and that these elite athletes are most likely to remain active at retirement, with the intensity and frequency of physical activity remaining above the average of the general population. This is supported by the fact that approximately half of retired Polish Olympic athletes become coaches, sports instructors or physical education teachers. The low number of observed deaths and the low statistical power were the predominant motivation to implement MLE, and not non-linear least squares. ${ }^{17}$ These MLE estimates suggest that healthy living and frequent exercise at the level of Olympic athletes decelerate the normal rate of ageing by $1 \%$ and reduce mortality risk threefold, and the possibility of an optimum benefit gained on the rate of ageing from frequent and intense physical training (table 2; Cohort I \& II athletes). Despite these apparent benefits, there can be negative health effects for elite athletes, evidenced by a high frequency of sports injuries and increased susceptibility to developing hypertrophic cardiomyopathy. In athletes, the most common physiological correlation among competitive athletes is cardiac size and sports performance. ${ }^{18}$ The enlargement of myocytes in athletes' hearts supports the elevated demand for energy output, and cardiac remodelling is seen as a crucial prerequisition to becoming an elite athlete, particularly for endurance sports. Such physiological adaptations and epigenetic changes among athletes are customised in accordance with individuals' environment and lifestyle habits. ${ }^{18-20}$ Therefore, longitudinal studies of elite athletes are highly suitable data sets to address the classic biological programmed senescence theories, for example, accumulation of damage, wear-and-tear and antagonistic pleiotropy. ${ }^{21} 22$

Theories on the accumulation of damage and the high frequency of wear-and-tear may support the theory of an accelerated failure time hazard or a higher rate of ageing among elite athletes. The inability to repair damages initiates a cascade of acute and chronic diseases. Thereby, the maximum damage-to-recovery shall hence be a one-time event, death. In addition, studies from evidence-based medicine have shown that an agespecific mortality rate requires a multifactorial risk assessment. ${ }^{23-25}$ Elite athletes are a highly selective group in the population, and we therefore expected to see a high degree of homogeneity. The lack of homogeneity could be explained by the diversity of their physical training and their likelihood of suffering different types of sports injuries, which could contribute to a variation in lifespan and susceptibility to less-common causes of death. For example, athletes engaged in sports requiring endurance and strength exhibit the greatest cardiac remodelling, and left-ventricle hypertrophic cardiomyopathy accounts for $36 \%$ deaths among young athletes. ${ }^{18}$ 26-28 This clinical observation supports the existence of potential trade-offs between lifespan and energy expenditure, ${ }^{22} 29$ and this subgroup of athletes requires a separate analysis for their survival profiles and are most certainly different from those on the Gompertzian baseline hazard list. The antagonistic pleiotropy theory by George C Williams may offer an explanation for why a small fraction of the population is more likely to excel in sports during early life, but experience an increase in susceptibility for rare causes of death and the early onset of rare diseases. ${ }^{27}{ }^{30}$ Approximately 20 genetic variants have been shown to be associated with sports requiring endurance and speed. ${ }^{31}$ For example, the ACTN-3 gene is a prime genetic factor for sports performance among sprinters, but affected carriers are more susceptible to developing left-ventricle cardiac hypertrophy and to experience premature death from sudden cardiac arrest. ${ }^{32}$ If trade-offs between sports performance and life span exist, we speculate an accelerated ageing process in affected carriers who are engaged in competitive sports, as these athletes are likely to experience death or cardiac-related health issues by their early $20 \mathrm{~s}$.

\section{CONCLUSION}

Our findings suggest that Polish elite athletes born from 1890-1919 experienced a slower rate of ageing, and had a lower risk for mortality and a longer life-expectancy than the general population from the same birth cohort. It is very unlikely that these survival benefits were gained within a short observational time. Therefore, we argue that participation in frequent sports from young adulthood reduces mortality risk, increases life-expectancy and slows the rate of ageing. However, our study suggests, with the exclusion of familial genetic 
diseases (eg, progeria) and instantaneous chemical exposures, the human rate of ageing appears to be quite robust. The age-specific mortality trajectories of Cohort I elite athletes also suggest frequent exercise can decelerate the rate of ageing by $1 \%$ with an achievement of threefold risk reduction in mortality. In comparison with those of the general population, the differences in energy expenditure, behavioural habits, body mass and sports expertise were likely to be the contributing factors to the higher variance in lifespan among elite athletes.

Mortality improvements since 1920 have changed individuals' susceptibilities for different causes of death, which has resulted in an increased variation in lifespan both in the general population and for elite athletes. Since our study had low statistical power, a meta-analysis on the mortality schedules for elite athletes would be a profitable avenue for future research. The development of a modified parametric frailty model that can account for a continuous reduction in mortality in life-table analyses would also be beneficial.

Acknowledgements Yuhui Lin would like to thank Adam Lenart for his invaluable guidance in R-programming. The authors would like to thank Owen Jones and the four reviewers for their comments. Previous appointment where the project was initiated: 1 Konrad-Zuse Strasse, Max Planck Institute for Demographic Research, 18057 Rostock, Germany.

Contributors $\mathrm{YL}$ designed and performed the statistical methodology and analysis, and drafted the manuscript. AG initiated the Polish Olympic longitudinal study and gained approved for the use of data and to conduct this study. AP assisted in the discussions of the study design and manuscript drafts. The authors have contributed to the intellectual content of the manuscript and have agreed on the final version to be published.

Funding This work was supported by the Max Planck Institute for Demographic Research and the Max Planck Society, Germany.

Competing interests None declared.

Ethics approval $A G$ and the Olympics Committee, Poland.

Provenance and peer review Not commissioned; externally peer reviewed.

Data sharing statement For access to the Polish Olympics athletes data set, contact AP. For conditioned data set and MLE estimations, contact YL.

Open Access This is an Open Access article distributed in accordance with the Creative Commons Attribution Non Commercial (CC BY-NC 4.0) license, which permits others to distribute, remix, adapt, build upon this work noncommercially, and license their derivative works on different terms, provided the original work is properly cited and the use is non-commercial. See: http:// creativecommons.org/licenses/by-nc/4.0/

\section{REFERENCES}

1. Peeters A, Backholer K. Is the health burden associated with obesity changing? Am J Epidemiol 2012;176:840-5.

2. Wijndaele $\mathrm{K}$, Brage $\mathrm{S}$, Besson $\mathrm{H}$, et al. Television viewing time independently predicts all-cause and cardiovascular mortality: the EPIC Norfolk study. Int J Epidemiol 2011;40:150-9.

3. Reilly JJ, Kelly J. Long-term impact of overweight and obesity in childhood and adolescence on morbidity and premature mortality in adulthood: systematic review. Int J Obes 2011;35:891-8.
4. Lee IM, Paffenbarger RS Jr. Associations of light, moderate, and vigorous intensity physical activity with longevity. The Harvard Alumni Health Study. Am J Epidemiol 2000;151:293-9.

5. Sarna S, Sahi T, Koskenvuo M, et al. Increased life expectancy of world class male athletes. Med Sci Sports Exerc 1993;25:237-44.

6. Coate D, Sun R. Survival estimates for elite male and female Olympic athletes and tennis championship competitors. Scand $J$ Med Sci Sports 2013;23:722-7.

7. Zwiers R, Zantvoord FWA, Engelaer FM, et al. Mortality in former Olympic athletes: retrospective cohort analysis. BMJ 2012;345: e7456.

8. Gompertz B. On the nature of the function expressive of the law of human mortality, and on a new mode of determining the value of life contingencies. Philos Trans R Soc Lond 1825;115:513-83.

9. Makeham WM. On the further development of Gompertz's law. J Inst Actuar 1889;28:152-9.

10. University of California, Berkeley (USA), and Max Planck Institute for Demographic Research (Germany). Human Mortality Database. 2012. http://www.mortality.org/

11. Gajewski AK, Poznańska A. Mortality of top athletes, actors and clergy in Poland: 1924-2000 follow-up study of the long term effect of physical activity. Eur J Epidemiol 2008;23:335-40.

12. Teramoto M, Bungum TJ. Mortality and longevity of elite athletes. J Sci Med Sport 2010;13:410-16.

13. Garatachea N, Santos-Lozano A, Sanchis-Gomar F, et al. Elite athletes live longer than the general population: a meta-analysis. Mayo Clin Proc 2014;89:1195-200.

14. Olshansky SJ, Carnes BA. Ever since Gompertz. Demography 1997;34:1-15.

15. Vaupel JW. Biodemography of human ageing. Nature 2010;464:536-42.

16. Sas AA, Snieder H, Korf J. Gompertz' survivorship law as an intrinsic principle of aging. Med Hypotheses 2012;78:659-63.

17. Petersen T. Estimating fully parametric hazard rate models with time-dependent covariates use of maximum likelihood. Sociol Methods Res 1986;14:219-46.

18. Maron BJ, Pelliccia A. The heart of trained athletes Circulation 2006;114:1633-44.

19. Rönn $\mathrm{T}$, Volkov $\mathrm{P}$, Davegårdh $\mathrm{C}$, et al. A six months exercise intervention influences the genome-wide DNA methylation pattern in human adipose tissue. PLoS Genet 2013;9:e1003572.

20. Pelliccia A, Maron BJ, Spataro A, et al. The upper limit of physiologic cardiac hypertrophy in highly trained elite athletes. N Engl J Med 1991;324:295-301.

21. Medawar PB. An unsolved problem of biology. HKLewis 1952;214:1-24.

22. Williams GC. Pleiotropy, natural selection, and the evolution of senescence. Evolution 1957;11:398-411.

23. Aalen OO. Heterogeneity in survival analysis. Stat Med 1988;7:1121-37.

24. Gerber Y, Myers V, Goldbourt U, et al. Long-term trajectory of leisure time physical activity and survival after first myocardial infarction: a population-based cohort study. Eur J Epidemiol 2011;26:109-16.

25. Yashin Al, Arbeev KG, Akushevich I, et al. Model of hidden heterogeneity in longitudinal data. Theor Popul Biol 2008;73:1-10.

26. Luijkx T, Cramer MJ, Prakken NH. Sport category is an important determinant of cardiac adaptation: an MRI study. Br J Sports Med 2012;46:1119-24.

27. Maron BJ, Roberts WC, McAllister HA, et al. Sudden death in young athletes. N Engl J Med 2003;349:1064-75.

28. Maron BJ, Doerer JJ, Haas TS, et al. Sudden deaths in young competitive athletes analysis of 1866 Deaths in the United States, 1980-2006. Circulation 2009;119:1085-92.

29. Kirkwood T, Value SE. The disposable soma theory of aging. Genet Eff Aging I/ 1990:1977-9.

30. Abel EL. Football increases the risk for Lou Gehrig's disease, amyotrophic lateral sclerosis. Percept Mot Ski 2007;104Pt 2):1251-4

31. Online Mendeline Inheritance in Man O. McKusick-Nathans Institute of Genetic Medicine. Baltimore, MD: John Hopkins University, 2014. http://omim.org/

32. Papadimitriou ID, Papadopoulos C, Kouvatsi A, et al. The ACTN3 gene in elite Greek track and field athletes. Int J Sports Med 2008;29:352-5 